\section{Reference values for ductus venosus doppler indices of normal gestation at 22-37 weeks: A Vietnamese cohort study}

Nguyen Thi Hong, ${ }^{1}$ Nguyen Minh Duc, ${ }^{2}$ Le Hoang, ${ }^{3}$ Phan Truong Duyet, ${ }^{3}$ Dang Hong Thien, ${ }^{3}$ Nguyen Thi Tuyet Mai, ${ }^{3}$ Hoang Thi Ngoc Tram, ${ }^{1}$ Ngo Minh Xuan, 2 Huynh Quang Huy 2

1Thai Nguyen University of Medicine and Pharmacy, Thai Nguyen; ${ }^{2}$ Pham Ngoc Thach University of Medicine, Ho Chi Minh City; ${ }^{3}$ National Hospital of Obstetrics and Gynecology, Ha Noi, Vietnam

\begin{abstract}
Prenatal doppler ultrasound of the ductus venosus plays an important role in the evaluation of fetal circulation and fetal heart function. We aimed to establish the percentile of Resistivity Index (RI) and Pulsatility Index (PI) of the Ductus Venosus (DV) among normal Vietnamese fetuses with a gestation from 22 to 37 weeks in a cohort study. Normal Vietnamese fetuses with a gestation ranging from 22 to 37 weeks were examined in a cohort study. The PI and RI were recorded from the DV. We analyzed 640 participants who all fulfilled the inclusion and exclusion criteria of our study. Results: There was a significant correlation between RI, PI and gestational age as shown with the equations $\mathrm{y}=0.077 \mathrm{x}-0.003 \mathrm{x}^{2}+$ $3.493 \mathrm{e}-5 \mathrm{x}^{3}(\mathrm{r}=0.97)$ and $\mathrm{y}=0.106 \mathrm{x}-$ $0.004 \mathrm{x}^{2}+4.5 \mathrm{e}-5 \mathrm{x}^{3}(\mathrm{r}=0.94)$, respectively. A centile module was constructed for the DV RI and PI indices among normal Vietnamese fetuses with gestation from 22 to 37 weeks.
\end{abstract}

\section{Introduction}

Screening and tracking during pregnancy, especially a high-risk pregnancy, is always an important task for obstetricians to ensure a safe and secure fetal outcome. It also helps to reduce infant mortality and improve maternal health. $1-4$

There are actually different methods of experimentation for determining the fetus' growth and safety status. Such modalities may identify irregularities in pregnancy early to trigger appropriate interventions that can save lives. ${ }^{5}$ Ultrasound is a non-invasive method commonly used in obstetrics and gynecology with many advantages. ${ }^{6-8}$ One of the most useful exploratory approaches nowadays is the use of vascular doppler ultrasound in the mother-child circulatory assessment. ${ }^{6}$ The Pulsatility Index (PI) and Resistivity Index (RI) of the Ductus Venosus (DV) have become a common ultrasonographic measurement during pregnancy. ${ }^{9}$ The importance of these doppler indices has been the topic of ongoing debate in the literature, and its reference value has not yet been identified in Vietnam among the normal population.

Doppler ultrasound has been widely used in obstetrics in Vietnam in recent years. A number of studies on the doppler indices have been conducted to evaluate umbilical arteries, cerebral arteries, uterine arteries during normal and abnormal pregnant periods. But so far no research on the DV doppler indexes has been performed in normal fetuses. The DV doppler indices for the Vietnamese population should be established in normal fetuses. This study aims to establish the percentile of DV RI and PI among normal fetuses with a gestation from 22 to 37 weeks in a Vietnamese cohort study.

\section{Materials and Methods}

\section{Patient population}

In this study a total of 640 women with normal singleton pregnancy were examined between the $22^{\text {nd }}$ and $37^{\text {th }}$ weeks of gestation. Fetal age is calculated from the last menstrual period and confirmed by the crown-rump length ultra-sonographic measurement. The following people were omitted from our study: women with gestational diabetes, preterm labor, antepartum congenital abnormalities, and maternal systemic diseases, women on a regimen of tocolytic and antihypertensive agents, and women for whom it is impossible to determine the exact gestational stage. The research was authorized by the Institutional Review Board at National Hospital of Obstetrics and Gynecology, Hanoi, Vietnam, and a written informed consent document was issued for each individual involved in the research.

\section{Ultrasonography}

A Voluson 730 Pr 4D colour Doppler ultrasound system (GE Healthcare, Milwaukee, WI, USA) was used to conduct all the ultrasonography procedures. Using an ultrasonic $3.5 \mathrm{MHz}$ frequency probe with color coded doppler and pulse doppler, the DV is a target of evaluation.

DV is positioned based on some steps.
Correspondence: Huynh Quang Huy, Department of Radiology, Pham Ngoc Thach University of Medicine. 2 Duong Quang Trung street, District 10, Ho Chi Minh City, Vietnam. E-mail: huyhq@pnt.edu.vn

Key words: Ductus venosus, Doppler ultrasound, Resistivity index, Pulsatility index.

Contributions: NTH and NMD contributed equally to this article as co-first authors. NTH and NMD gave a substantial contribution in acquisition, analysis, and data interpretation. NMD and HQH prepared, drafted, and revised manuscript critically for important intellectual content. Each author gave the final approval of the version to be published and agreed to be accountable for all aspects of the work, ensuring that questions related to the accuracy or integrity of any part of the work are appropriately investigated and resolved.

Conflicts of interest: The authors declare no conflict of interest.

Funding: None.

Ethical approval and informed consent: Institutional Review Board at National Hospital of Obstetrics and Gynecology (Hanoi, Vietnam) approved this prospective study. Written informed consent of patients was obtained.

Received for publication: 10 March 2020.

Accepted for publication: 13 May 2020

This work is licensed under a Creative Commons Attribution NonCommercial 4.0 License (CC BY-NC 4.0).

(C) Copyright: the Author(s), 2020

Licensee PAGEPress, Italy

Pediatric Reports 2020; 12:8535

doi:10.4081/pr.2020.8535

We initially trace the horizontal segment along the fetal spine and then we see the aorta that descends. The anterior thorax is the right fetal heart ventricle in front of the aorta that leads to the inferior vena cava. The most prominent landmark is the umbilical vein that flows into the fetal liver. We may find a branch from the umbilical vein into the lower vena cava, which is the DV (Figure 1 ), by the umbilical vein.

Criteria of appropriate range to calculate indices were: when five doppler spectra are reached on the screen drift band, stop for analysis and measurement indices; mark the maximum velocity of the systolic wave (S) and the maximum velocity of the diastolic wave (D) and the minimum endpoint of the diastolic wave (a); In the ultrasound machine, the indices are automatically determined 
when the ruler is set to the highest point of the S, D, a waves; conduct a second revision of the measurements, taking the mean of the two measurements as the data to be obtained in the test. The DV doppler indices are used in the study (Figure 2): Pulsatility index (PI): PI $=\mathrm{S}-\mathrm{a} / \mathrm{TAMX}$, Resistivity index $(\mathrm{RI}): \mathrm{RI}=\mathrm{S}-\mathrm{a} / \mathrm{S}$.

\section{Statistical analysis}

Data were analyzed using the software SPSS for Windows, version 15.0 (SPSS Inc., Chicago, IL, USA). Analyze the developmental rules of the mean values of the DV doppler indices by the method of calculating the correlation between two quantities for each function $\mathrm{y}=\mathrm{f}(\mathrm{x})$ where $\mathrm{y}$ is the doppler index, $\mathrm{x}$ is gestational age, with correlation when $r>0.5$ and correlated closely when $r>0.7$. Based on the correlation coefficient, the data table established, the upper and lower limits of each doppler index were determined using 5\%, 10\%, 50\%, 95\%, and $97 \%$ centile curves for each doppler index by gestational age.

\section{Results}

A total of 640 qualified participants meeting the inclusion and exclusion requirements were evaluated during the period June 2014 to January 2017 at the National Obstetrics and Gynecology Hospital. The crude PI mean was relatively stable and marginally decreased with gestation of 22 to 37 weeks. In order to prove and find out the PI development rules from the week of 22-37, we determined the correlation between y (PI) andx (gestational age) for each of the first, second, and third order functions, to decide the function with the highest correlation coefficient representing proper development rules. Solution of the third-order function chosen after replacing $\mathrm{x}$ with gestational age will obtain the mean PI values per gestational age shown in Table 1.

We implemented Table 2 on the basis of the formula for calculating the values corresponding to the centile lines and draw the following diagram for clinical use (Figure 3).

Similar to the calculation of the PI, the functions selected for calculating the centile lines for the RI from 22 to 37 weeks are the third order function $\mathrm{y}=0.077 \mathrm{x}-0.003 \mathrm{x}^{2}+$ $3.493 e-5 x^{3}$.

The chosen variable rule calculates the mean values and the corresponding values for the centile lines from the third order function: $3,5,10,50,90,95,97$ as shown in Table 3. We drew the DVPI centile curve for gestational age from 22-37 weeks from the

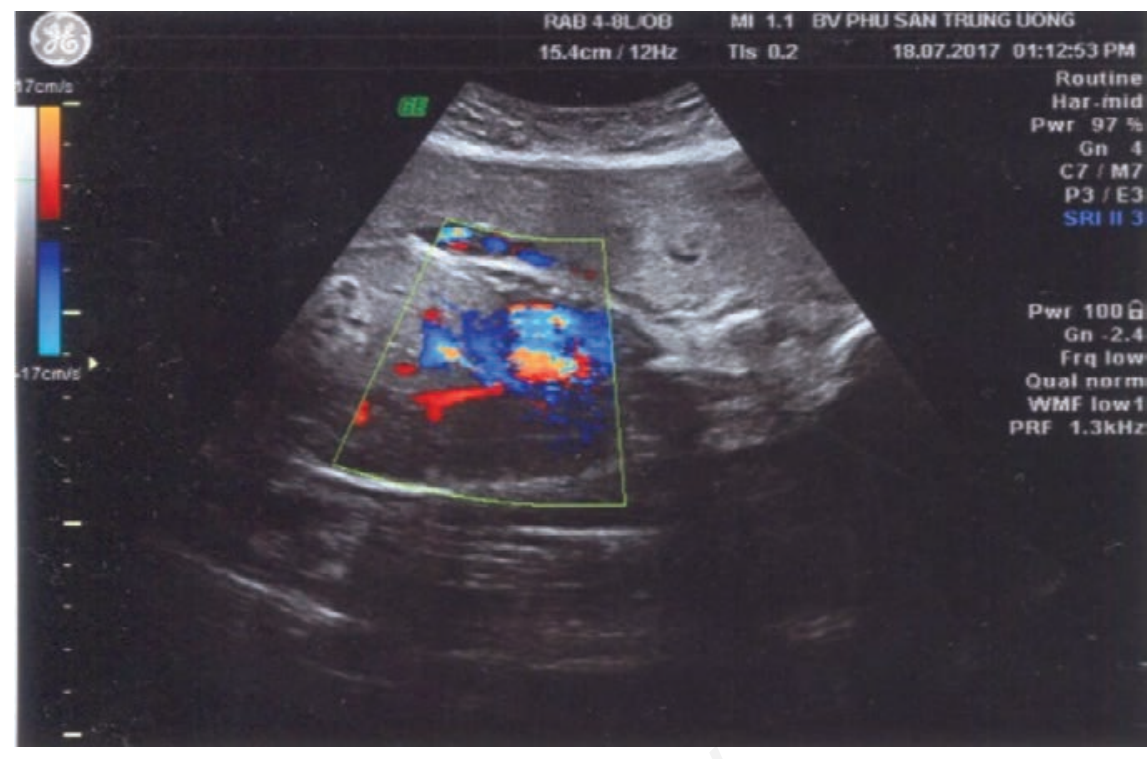

Figure 1. Imaging of DV located by color doppler.

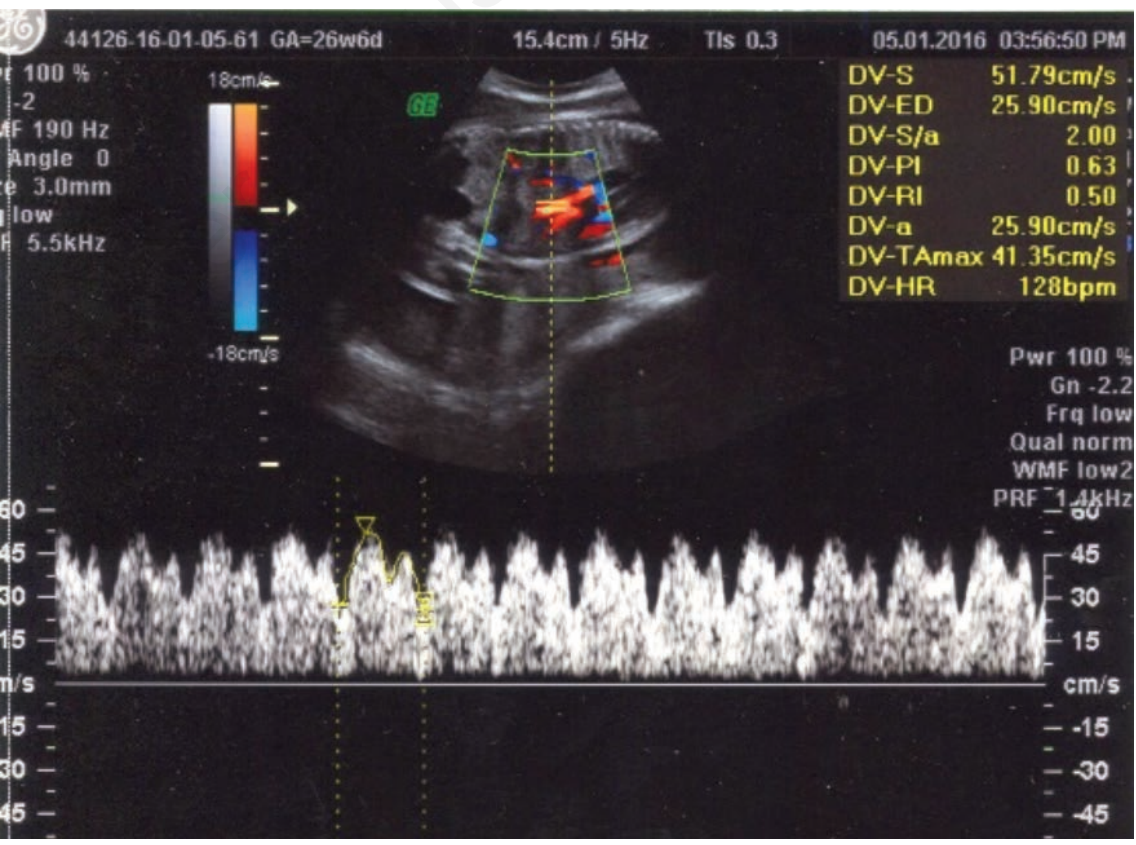

Figure 2. Imaging of normal DV doppler measurement.

table above (Figure 4).

Table 1. The $y(P I)$ to $x$ (gestational age) association.

\begin{tabular}{lll} 
Function & Equation & $r$ \\
First order & $\mathrm{y}=0.025 \mathrm{x}$ & 0.79 \\
Second order & $\mathrm{y}=0.067 \mathrm{x}-0.001 \mathrm{x}^{2}$ & 0.88 \\
\hline Third order & $\mathrm{y}=0.106 \mathrm{x}-0.004 \mathrm{x}^{2}+4.5 \mathrm{e}-5 \mathrm{x}^{3}$ & 0.90 \\
\hline
\end{tabular}




\section{Discussion}

The DV has an operation like a sphincter. It is a small vessel transmitting a high-velocity jet later to re-change the foramen ovale. The high peak velocity in the DV, compareble with arterial velocities, probably gives enough momentum to the blood to replenish the foramen ovale without extensive mixing with the deoxygenated blood. ${ }^{10,11}$ DV velocimetry brings new diagnostic possibilities for preload or heart function assessment. The typical doppler flow waveform of the ductus venosus shows the continuous flow of the triphasic forware throughout the cardiac cycle with a peak during the systole, another one during the diastolic filling, and an atrial nadir during the contrast. ${ }^{12,13}$ Many research investigated the diagnostic importance of ductus venosus blood flow in the diagnosis of fetuses with congenital heart disease, hypoxic or congestive fetal myocardial diseases from both a clinical and a scientific viewpoint. There is now a welldocumented link between irregular DV flow, chromosomal anomalies, and adverse fetal outcomes. ${ }^{14-16}$ Consequently, the purpose of this study was to create longitudinal references for RI, PI, and suitable diameter for use with serial measurements for fetal surveillance, and we have also provided the necessary terms for individual conditional reference intervals suitable for individual serial measurements.

The fetal movement was often examined with the DVPI. According to our results, the DVPI was closely correlated with gestational age according to a third-order feature in typical pregnancies from 22 to 37 weeks. Some authors developed a PI reduction chart based

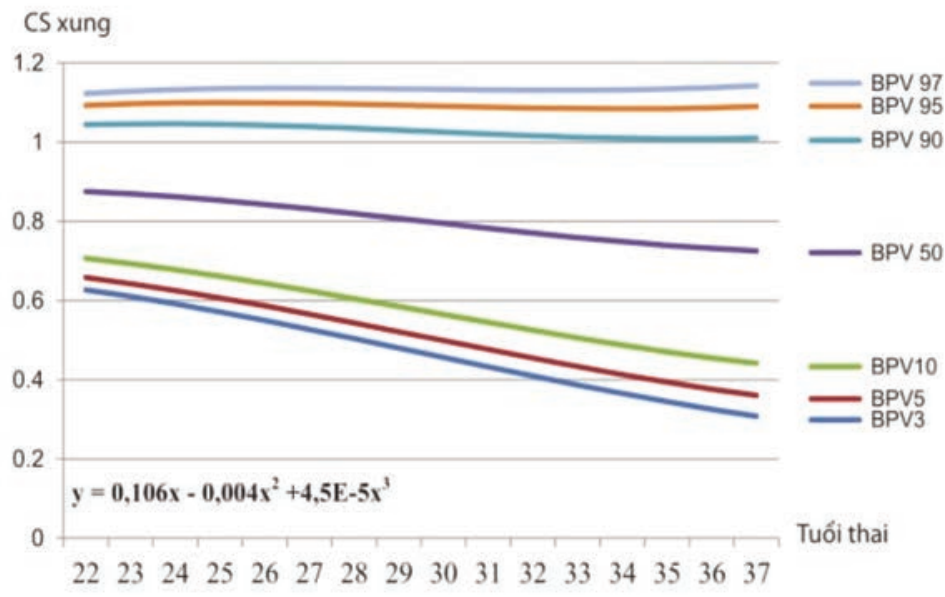

Figure 3. DVPI centile diagram for gestational age 22-37 weeks.

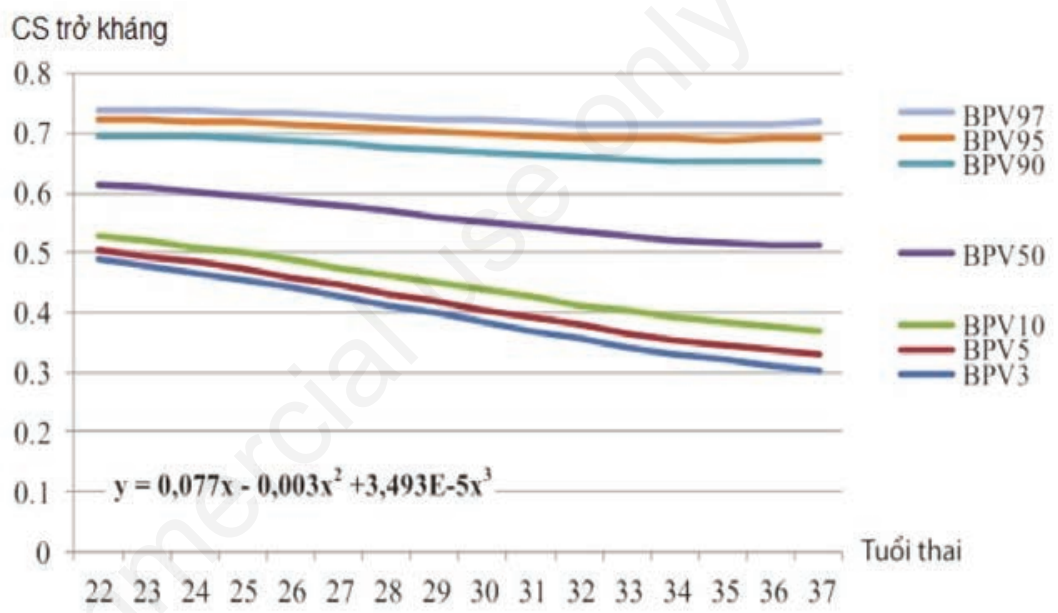

Figure 4. DVRI centile diagram for gestational age 22-37 weeks.

Table 2. PI values corresponding to centile lines 3, 5, 10, 50, 90, 95, 97 for 22-37 weeks of gestational age.

\begin{tabular}{|c|c|c|c|c|c|c|c|c|c|}
\hline \multirow{2}{*}{ Gestational age } & \multirow[t]{2}{*}{$\mathbf{N}$} & \multirow[t]{2}{*}{ SD } & \multicolumn{7}{|c|}{ Distribution of PI according to centile lines } \\
\hline & & & $3 \%$ & $5 \%$ & $10 \%$ & $50 \%$ & $90 \%$ & $95 \%$ & $97 \%$ \\
\hline 22 & 54 & 0.13 & 0.63 & 0.66 & 0.71 & 0.88 & 1.04 & 1.09 & 1.12 \\
\hline 23 & 49 & 0.14 & 0.61 & 0.64 & 0.69 & 0.87 & 1.05 & 1.10 & 1.13 \\
\hline 24 & 41 & 0.14 & 0.59 & 0.63 & 0.68 & 0.86 & 1.05 & 1.10 & 1.13 \\
\hline 25 & 38 & 0.15 & 0.57 & 0.61 & 0.66 & 0.85 & 1.05 & 1.10 & 1.14 \\
\hline 26 & 40 & 0.16 & 0.55 & 0.59 & 0.64 & 0.84 & 1.04 & 1.10 & 1.14 \\
\hline 27 & 45 & 0.16 & 0.53 & 0.57 & 0.62 & 0.83 & 1.04 & 1.10 & 1.14 \\
\hline 28 & 42 & 0.17 & 0.50 & 0.54 & 0.60 & 0.82 & 1.03 & 1.10 & 1.14 \\
\hline 29 & 40 & 0.17 & 0.48 & 0.52 & 0.58 & 0.81 & 1.03 & 1.09 & 1.13 \\
\hline 30 & 39 & 0.18 & 0.46 & 0.50 & 0.56 & 0.80 & 1.03 & 1.09 & 1.13 \\
\hline 31 & 42 & 0.19 & 0.43 & 0.48 & 0.54 & 0.78 & 1.02 & 1.09 & 1.13 \\
\hline 32 & 50 & 0.19 & 0.41 & 0.45 & 0.52 & 0.77 & 1.02 & 1.09 & 1.13 \\
\hline 33 & 35 & 0.20 & 0.39 & 0.43 & 0.51 & 0.76 & 1.01 & 1.08 & 1.13 \\
\hline 34 & 32 & 0.20 & 0.37 & 0.41 & 0.49 & 0.75 & 1.01 & 1.08 & 1.13 \\
\hline 35 & 32 & 0.21 & 0.34 & 0.39 & 0.47 & 0.74 & 1.01 & 1.08 & 1.13 \\
\hline 36 & 31 & 0.22 & 0.33 & 0.38 & 0.46 & 0.73 & 1.01 & 1.09 & 1.14 \\
\hline 37 & 30 & 0.22 & 0.31 & 0.36 & 0.44 & 0.73 & 1.01 & 1.09 & 1.14 \\
\hline
\end{tabular}


Table 3. RI values corresponding to the centile lines from 22-37 weeks: 3, 5, 10, 50, 90, 95, 97 for gestational age.

\begin{tabular}{|c|c|c|c|c|c|c|c|c|c|}
\hline \multirow[t]{2}{*}{ GA } & \multirow[t]{2}{*}{$\mathbf{N}$} & \multirow[t]{2}{*}{ SD } & \multicolumn{7}{|c|}{ Distribution of RI bv centile lines } \\
\hline & & & $3 \%$ & $5 \%$ & $10 \%$ & $50 \%$ & $90 \%$ & $95 \%$ & $97 \%$ \\
\hline 22 & 54 & 0.07 & 0.49 & 0.51 & 0.53 & 0.61 & 0.70 & 0.72 & 0.74 \\
\hline 23 & 49 & 0.07 & 0.48 & 0.50 & 0.52 & 0.61 & 0.70 & 0.72 & 0.74 \\
\hline 24 & 41 & 0.07 & 0.47 & 0.48 & 0.51 & 0.60 & 0.70 & 0.72 & 0.74 \\
\hline 25 & 38 & 0.08 & 0.45 & 0.47 & 0.50 & 0.60 & 0.69 & 0.72 & 0.74 \\
\hline 26 & 40 & 0.08 & 0.44 & 0.46 & 0.49 & 0.59 & 0.69 & 0.72 & 0.73 \\
\hline 27 & 45 & 0.08 & 0.43 & 0.45 & 0.48 & 0.58 & 0.68 & 0.71 & 0.73 \\
\hline 28 & 42 & 0.08 & 0.41 & 0.43 & 0.46 & 0.57 & 0.68 & 0.71 & 0.73 \\
\hline 29 & 40 & 0.09 & 0.40 & 0.42 & 0.45 & 0.56 & 0.67 & 0.71 & 0.73 \\
\hline 30 & 39 & 0.09 & 0.38 & 0.41 & 0.44 & 0.55 & 0.67 & 0.70 & 0.72 \\
\hline 31 & 42 & 0.09 & 0.37 & 0.39 & 0.43 & 0.54 & 0.66 & 0.70 & 0.72 \\
\hline 32 & 50 & 0.10 & 0.36 & 0.38 & 0.41 & 0.54 & 0.66 & 0.69 & 0.72 \\
\hline 33 & 35 & 0.10 & 0.34 & 0.37 & 0.40 & 0.53 & 0.66 & 0.69 & 0.72 \\
\hline 34 & 32 & 0.10 & 0.33 & 0.36 & 0.39 & 0.52 & 0.65 & 0.69 & 0.71 \\
\hline 35 & 32 & 0.11 & 0.32 & 0.34 & 0.38 & 0.52 & 0.65 & 0.69 & 0.72 \\
\hline 36 & 31 & 0.11 & 0.31 & 0.34 & 0.38 & 0.51 & 0.65 & 0.69 & 0.72 \\
\hline 37 & 30 & 0.11 & 0.30 & 0.33 & 0.37 & 0.51 & 0.65 & 0.69 & 0.72 \\
\hline
\end{tabular}

on the first, second-order functions such as Tongprasert et al., 17 Bahlmann et al., 18 AxtFliedner et al. ${ }^{19}$ The authors had a common remark that the DVPI was associated with gestational age and given various correlation functions based on race of population. The authors set a table of reference values for the DV doppler index for different countries from the correlation equation. There are several explanations for this difference: gestational age, methods of analysis, methods of processing data and ethnicity of the population. From the literature review, the authors found that the DVPI in typical pregnancies decreased with gestational age that properly reflected placental circulation physiology. ${ }^{20,21}$ As the placental papilla grew, the capillary system was broad, the blood vessels were wide, the cell culture layers at the end of pregnancy were gradually thin to facilitate the metabolism between mother and fetus, and help the baby grow well. The fetal DVPI had however the features of different racial groups. ${ }^{22,23}$ Hence it is necessary to develop the physiological constants of the DVPI in different countries for each population group. In our research the RI was closely correlated with gestational age in average pregnant women at 22-37 weeks of gestation. Compared with other authors in the world, the correlation coefficient between RI and gestational age showed that the majority of authors selected a first-order function like Tongprasert et al,17 Bahlmann et al. ${ }^{18}$ Comparison of the correlation function between the RI and the gestational age of the authors worldwide revealed that the authors developed an RI histogram for gestational age with the functions of the first and second order. ${ }^{24-26}$ We chose the third-order function in this analysis because we noticed that the thirdorder function had a higher correlation coefficient and suggested that RI's variable law decreased more reliably with an increase in gestational age. Differences in correlation functions may be due to different authors " gestational age at the start of the study, method of study, method of data analysis.

Our study results were likewise compatible with some other writers in the world. ${ }^{27,28}$ DVRI value decreased steadily as gestational age increased, indicating the RI of decreased placental circulation, suggesting easy maternal and fetal metabolism was facilitated by circulation in the placental papilla. It facilitated the fetus' development without the risk of retarding the uterus' fetal production. Therefore, the risk to the fetus was very small, when the DVRI was normal. Studies have shown that the DVRI is different and has features across different racial groups. Therefore it is important for each population race to establish physiological constants of DVPI.

\section{Conclusions}

Ductus venosus doppler indices may constantly decrease with an increase in the age of gesture. The need to set normal range is therefore of great importance. A centile module was constructed for the ductus venosus RI and PI indices among normal Vietnamese fetuses with gestation from 22 to 37 weeks. Further studies should perform and validate our findings.

\section{References}

1. Greco A, Ragucci M, Liuzzi R, et al. Noninvasive Ultrasound Monitoring of Embryonic and Fetal Development in Chinchilla lanigera to Predict Gestational Age: Preliminary Evaluation of This Species as a Novel Animal Model of Human Pregnancy. Contrast Media Mol Imaging 2019:6319476.

2. Haws RA, Yakoob MY, Soomro T, et al. Reducing stillbirths: screening and monitoring during pregnancy and labour. BMC Pregnancy Childbirth 2009;9:S5

3. Scully S, Evans AC, Carter F, et al. Ultrasound monitoring of blood flow and echotexture of the corpus luteum and uterus during early pregnancy of beef heifers. Theriogenol 2015;83:44958.

4. Vinayagam D, Bowe S, Sheehan E, et al. Non-Invasive Haemodynamic Monitoring in Pregnancy: A Comparative Study Using Ultrasound and Bioreactance. Fetal Diagn Ther 2017;41:273-282.

5. Hui L. Noninvasive Approaches to Prenatal Diagnosis: Historical Perspective and Future Directions. Methods Mol Biol 2019;1885:45-58.

6. Campbell S. A short history of sonography in obstetrics and gynaecology. Facts Views Vis Obgyn 2013;5:213-29.

7. Messawa M, Ma'ajeni E, Daghistani $\mathrm{MH}$, et al. The role of doppler ultrasound in high risk pregnancy: A comparative study. Niger Med J 2012;53:116-20. 
8. York AB, Bagwell LL. Pregnancy Choices and the Role of Ultrasound Viewing. J Christ Nurs 2019;36:54-7.

9. Kalayci H, Yilmaz Baran S, Dogan Durdag G, et al. Reference values of the ductus venosus pulsatility index for pregnant women between 11 and $13(+6)$ weeks of gestation. J Matern Fetal Neonatal Med 2020;33:1134-9.

10. Kiserud T. Hemodynamics of the ductus venosus. Eur J Obstet Gynecol Reprod Biol 1999;84:139-47.

11. Kiserud T. Fetal venous circulation - an update on hemodynamics. J Perinat Med 2000;28:90-6.

12. Baschat AA. Relationship between placental blood flow resistance and precordial venous Doppler indices. Ultrasound Obstet Gynecol 2003;22:561-6.

13. Bellotti M, Pennati G, De Gasperi C, et al. Simultaneous measurements of umbilical venous, fetal hepatic, and ductus venosus blood flow in growthrestricted human fetuses. Am J Obstet Gynecol 2004;190:1347-58.

14. Ebbing C, Rasmussen S, Godfrey KM, et al. Redistribution pattern of fetal liver circulation in intrauterine growth restriction. Acta Obstet Gynecol Scand 2009;88:1118-23.

15. Frusca T, Todros T, Lees C, et al. Outcome in early-onset fetal growth restriction is best combining computerized fetal heart rate analysis with ductus venosus Doppler: insights from the Trial of Umbilical and Fetal
Flow in Europe. Am J Obstet Gynecol 2018;218:S783-S789.

16. Tchirikov M, Schroder HJ, Hecher K. Ductus venosus shunting in the fetal venous circulation: regulatory mechanisms, diagnostic methods and medical importance. Ultrasound Obstet Gynecol 2006;27:452-61.

17. Tongprasert F, Srisupundit K, Luewan S, et al. Normal reference ranges of ductus venosus Doppler indices in the period from 14 to 40 weeks' gestation. Gynecol Obstet Invest 2012;73:32-7.

18. Bahlmann F, Wellek S, Reinhardt I, et al. Reference values of ductus venosus flow velocities and calculated waveform indices. Prenat Diagn 2000;20:623-34.

19. Axt-Fliedner R, Wiegank U, Fetsch C, et al. Reference values of fetal ductus venosus, inferior vena cava and hepatic vein blood flow velocities and waveform indices during the second and third trimester of pregnancy. Arch Gynecol Obstet 2004;270:46-55.

20. DeVore GR, Horenstein J. Ductus venosus index: a method for evaluating right ventricular preload in the secondtrimester fetus. Ultrasound Obstet Gynecol 1993;3:338-42.

21. Rizzo G, Capponi A, Arduini D, et al. Ductus venosus velocity waveforms in appropriate and small for gestational age fetuses. Early Hum Dev 1994;39:15-26.

22. Audette MC, Kingdom JC. Screening for fetal growth restriction and placental insufficiency. Semin Fetal Neonatal Med 2018;23:119-25.
23. Krishna U, Bhalerao S. Placental insufficiency and fetal growth restriction. J Obstet Gynaecol India 2011;61:505-11.

24. Chen LK, Yang YM, Yang YH, et al. Doppler measurement of the changes of fetal umbilical and middle cerebral artery velocimetric indices during continuous epidural labor analgesia. Reg Anesth Pain Med 2011;36:249-255.

25. Morales Rosello J, Hervas Marin D, Perales Marin A, et al. Doppler study of the fetal vertebral and middle cerebral arteries in fetuses with normal and increased umbilical artery resistance indices. J Clin Ultrasound 2013;41:2249.

26. Srikumar S, Debnath J, Ravikumar R, et al. Doppler indices of the umbilical and fetal middle cerebral artery at 18-40 weeks of normal gestation: A pilot study. Med J Armed Forces India 2017;73:23241.

27. Hsu TY, Hsu JJ, Fu HC, et al. The changes in Doppler indices of fetal ductus venosus and umbilical artery after amnioinfusion for women with preterm premature rupture of membranes before 26 weeks' gestation. Taiwan J Obstet Gynecol 2009;48: 268-72.

28. Hsu TY, Ou CY, Chang SY, et al. Waveforms of the ductus venosus blood flow in normal human fetuses aged 8-38 weeks. Chang Gung Med J 2001;24:71723. 